\title{
A Material Model Driver for DYNA3D
}

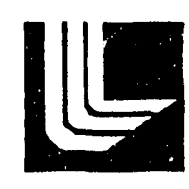

John O. Hallquist

Consultant

\author{
Robert G. Whirley \\ Methods Development Group \\ Mechanical Engineering
}

Manuscript date:

February 22, 1990 


\title{
A Material Model Driver for DYNA3D
}

\author{
John O. Hallquist \\ Consultant \\ Robert G. Whirley \\ Methods Development Group \\ Mechanical Engineering
}

February 22, 1990

\begin{abstract}
This report describes a material model driver which has recently been implemented in the DYNA3D code. The material model driver allows plotting of the constitutive response predicted by a material model under a given load path. This capability is particularly useful when fitting complex material models to experimental data. The plotting capability of the material model driver facilitates comparison of the simulated material stress-strain behavior with actual material test results.
\end{abstract}




\section{Introduction}

A material model driver has recently been implemented in the DYNA3D explicit finite element code. This driver allows calculation of the material constitutive response to a specified load path without including the effects of dynamic response. When the material model driver is used, the dynamic equations of motion are not applied; thus the material behavior is simulated independent of inertial effects. Rate dependence in the constitutive model is properly treated, however.

\section{Input Definition}

The material model driver is invoked by setting the total number of beam, shell, and solid elements to zero in a standard DYNA3D input file. The number of load curves should be set to nine, and the termination time should be set to the desired length of the driver run. The complete state dump interval is interpreted as the time step to be used in the material model driver run. Plotting information is saved for every step of a driver run. The driver run may contain a maximum of 100 steps in the present implementation.

The remainder of the input file skould be specified as in the DYNA3D User's Manual [1] up through the material definition. Immediately after the material definition, nine load curves describing the strain path should be defined. These nine curves describe the time history of the displacement gradient components shown in Table 1.

Table 1 - Load Curve Definitions

\begin{tabular}{|c|c|}
\hline Load Curve Number & Component Definition \\
\hline \hline 1 & $\frac{\partial u}{\partial r}$ \\
\hline 2 & $\frac{\partial v}{\partial y}$ \\
\hline 3 & $\frac{\partial w}{\partial v}$ \\
\hline 4 & $\frac{\partial u}{\partial y}$ \\
\hline 5 & $\frac{\partial v}{\partial x}$ \\
\hline 6 & $\frac{\partial u}{\partial z}$ \\
\hline 7 & $\frac{\partial w}{\partial x}$ \\
\hline 8 & $\frac{\partial v}{\partial v}$ \\
\hline 9 & $\frac{\partial w}{\partial y}$ \\
\hline
\end{tabular}


The rate of deformation is found by taking the symmetric part of a finite difference time derivative of the above components. For example, if the above components are considered to form a tensor $\mathbf{S}$, then a tensor $\mathbf{L}$ is calculated from

$$
\mathbf{L}(t)=\frac{\mathbf{S}(t)-\mathbf{S}\left(t_{k-1}\right)}{\left(t-t_{k}\right)}
$$

and then the rate of deformation is found from

$$
\mathrm{d}=\frac{1}{2}\left(\mathbf{L}+\mathbf{L}^{T}\right)
$$

and the spin is

$$
\omega=\frac{1}{2}\left(\mathbf{L}-\mathbf{L}^{T}\right)
$$

In an infinitesimal strain context, these quantities may be interpreted as given in Table 2 .

Table 2 - Small Strain Load Curve Interpretations

\begin{tabular}{|c|c|}
\hline Load Curve Number & Small Strain Interpretation \\
\hline \hline 1 & $\varepsilon_{x x}$ \\
\hline 2 & $\varepsilon_{y y}$ \\
\hline 3 & $\varepsilon_{z z}$ \\
\hline 4 & $\varepsilon_{x y}$ \\
\hline 5 & $\varepsilon_{y x}$ \\
\hline 6 & $\varepsilon_{x z}$ \\
\hline 7 & $\varepsilon_{z x}$ \\
\hline 8 & $\varepsilon_{y z}$ \\
\hline 9 & $\varepsilon_{z y}$ \\
\hline
\end{tabular}

\section{Interactive Commands}

The DYNA3D material model driver contains an integral plotting package to allow immediate display of simulation results. After reading the input file and completing the calculations, DYNA3D gives a command prompt to the terminal. A summary of the available interactiv. commands is given below. An online help package is available by typing help at the prompt.

ASCL - scale all abscissa data by $f$. default is $f=1$ 
OSCL - scale all ordinate data by $f$. default is $f=1$

ASET -amin omax - set min and max values on abscissa to amin and amax, respectively. if $\operatorname{amin}=\operatorname{amax}=0$, scaling is automatic.

OSET - omin omax - set min and max values on ordinate to omin and omax, respectively. If omin $=$ omax $=0$, scaling is automatic.

GRID - tmds or graphics displays will be overlayed by by a grid of orthogonal lines

NOGRID - tmds or graphics displays will not be overlayed by a grid of orthogonal lines

LOGO - puts llnl logo on all plots (default). Retyping this command removes the logo.

TV $\mathrm{n}$ - use tmds $\mathrm{n}$ or graphics device $\mathrm{n}$ where $\mathrm{n}$ is the monitor or device number

TV - $\mathrm{n} 1 \mathrm{n} 2 \mathrm{n} 3$ - use color tmds with monitor numbers $\mathrm{n} 1, \mathrm{n} 2$, and $\mathrm{n} 3$ for red, green, and blue channels, respectively.

PLOTS box ann - create a plotfile for box number ann that contains a record of the timds display

CLASS lev - reset classification level of hardcopy output from default unclassified to: proglev, pard, adp, confidnt, srd, or system. This command must precede the plots command if used.

RJET $n$ - send a copy of the fr 80 file to rjet $n$

TIME $\mathrm{c}$ - plot component $\mathrm{c}$ versus time

CROSS c1 c2 - plot component c1 versus c2

PRINT - print plotted time history data in file "pamper." Only data plotted after this command is printed. File name can be changed with the "file" command.

FILE name - change pampers filenaine to name. 
RDLC $\mathrm{m} n \mathrm{r} 1 \mathrm{z} 1 \ldots \mathrm{m} \mathrm{zn}$ - redefine load curve $\mathrm{m}$ using $\mathrm{n}$ coordinate pairs $(\mathrm{r} 1, \mathrm{z} 1),(\mathrm{r} 2, \mathrm{z} 2), \ldots,(\mathrm{rn}, \mathrm{zn})$

CONTINUE - reanalyze material model

ECOMP - display component numbers on the tmds or graphics display

$1 \mathrm{x}$-stress

$2 y$-stress

$3 z$-stress

$4 \mathrm{xy}$-stress

5 yz-stress

$6 \mathrm{zx}$-stress

7 effective plastic strain

8 pressure

9 von Misrs (effective) stress

101 st principal deviatoric stress

11 2nd principal deviatoric stress

12 3rd principal deviatoric stress

13 maximum shear stress

141 st principal stress

15 2nd principal stress

16 3rd principal stress

$17 \ln (\mathrm{v} / \mathrm{v} 0)$

18 relative volume

$19 \mathrm{v} 0 / \mathrm{v}-1.0$

201 st history variable

21 2nd history variable

Adding 100 or 400 to component numbers 1-16 yields strains and strain rates, respectively.

CHGL $\mathrm{n}$ - change label for component $\mathrm{n}$. DYNA3D prompts for new label. 
QUIT, END, T - exit the material model driver program

Presently, the material model driver is implemented only for solid element material models. Specifically, the driver does not support material models for shell elements or beam elements. Extension of the driver to these areas is planned for the near future.

\section{Examples}

The first example is a simple linear elastic material (model 1) subjected to uniaxial strain. The complete DYNA3D input file for this example is given in Appendix A. Figures 1-3 were generated using the CROSS comminand, and show the Cauchy stress - infinitesimal strain curves generated by the material model driver. For this example, a Young's modulus of $30.0 \times 10^{6}$ and a Poisson's ratio of .25 were used. From Figure 1, it is easily verified that the slope of the line is $\lambda+2 G=3.6 \times 10^{7}$, where $\lambda$ is the Lame parameter and $G$ is the shear modulus. Figures 2 and 3 show the transverse Cauchy stresses plotted against axial strain, and the slope is seen to be $\lambda$, as expected. Thus, this simple example has illustrated the agreement between elasticity theory and the DYNA3D elastic material model for the case of uniaxial strain.

The second example considers a geologic material represented by the Soil and Crushable Foam (model 5) in DYNA3D. The DYNA3D input file for this example is given in Appendix B. The parameter values are taken from an actual soil model used for production calculations, and are shown in Table 3. The pressure-volume curve is described in Table 4.

Table 3 - Model 5 Parameter Values

\begin{tabular}{|c|c|}
\hline Parameter & Value \\
\hline \hline shear modulus & $6.70 \times 10^{5}$ \\
\hline bulk modulus & $4.20 \times 10^{6}$ \\
\hline$a_{0}$ & $9.90 \times 10^{6}$ \\
\hline$a_{1}$ & $3.50 \times 10^{3}$ \\
\hline$a_{2}$ & 0.30 \\
\hline pressure cutoff & $-5.70 \times 10^{2}$ \\
\hline
\end{tabular}


Table 4 - Pressure-Volume Curve for Soil Model

\begin{tabular}{|c|c|}
\hline Relative Volume $\ln \left(\frac{\nu}{v_{0}}\right)$ & Pressure \\
\hline \hline 0.0 & 0.0 \\
\hline$-4.0 \times 10^{-3}$ & $2.9 \times 10^{3}$ \\
\hline$-1.0 \times 10^{-2}$ & $8.7 \times 10^{3}$ \\
\hline$-1.6 \times 10^{-2}$ & $1.8 \times 10^{3}$ \\
\hline$-2.0 \times 10^{-2}$ & $2.5 \times 10^{4}$ \\
\hline$-2.6 \times 10^{-2}$ & $3.5 \times 10^{4}$ \\
\hline$-3.0 \times 10^{-2}$ & $4.1 \times 10^{4}$ \\
\hline$-3.7 \times 10^{-2}$ & $5.3 \times 10^{4}$ \\
\hline$-4.0 \times 10^{-2}$ & $5.8 \times 10^{4}$ \\
\hline
\end{tabular}

The prescribed strain history is a shear deformation in the $x-y$ direction, increasing linearly from zero to a strain of $5 \%$ at a time of 100 . Superimposed on this shear deformation is a volumetric compaction starting at a time of 50.0 , and increasing linearly to a volum.tric strain of -0.03 at a time of 100. Thus, the first half of the simulation illustrates the material response at zero pressure, while the second half of the simulation shows the effect of increasing pressure on the shear behavior. Figure 4 shows the resulting plot of shear stress vs. pressure, and Figure 5 shows the pressure-volume behavior predicted by the model. Figure 6 directly shows the influence of pressure on the shear stress.shear strain curve. Clearly, plots such as these can be easily correlated with experimental data to determine the appropriate parameters for a DYNA3D material model. 

$\begin{array}{lll}1 & 2\end{array}$
$100.0000 \quad 0.000000$
82
$100.0000 \quad 0.000000$
92
$100.0000 \quad 0.000000$

\section{Appendix B: DYNA3D Input for Example 2}

material model 5 driver example 1 soil shear

1

9

$100.0000 \quad 0.0 \quad .1000000$

$151.000 e+00$

Sidewinder Tuff \#STBNM1 units=psi,sec (DYNA3D)

$6.700 e+054.200 e+069.900 e+06 \quad 3.500 e+03 \quad 3.000 e-01-5.700 e+02$

0 . $0 . \quad-4.000 e-032.900 e+03$

$-1.000 e-028.700 e+03-1.600 e-021.800 e+04$

$-2.000 e-022.500 e+04-2.600 e-023.500 e+04$

$-3.000 e-024.150 e+04-3.700 e-02 \quad 5.300 e+04$

$-4.000 e-02 \quad 5.800 e+04-8.300 e-02 \quad 1.300 e+05$

$\begin{array}{rrr}1 & 3 & \\ & 0.0 & 0.0 \\ & 50.0 & 0.0 \\ 100.000 & -.0100 \\ 2 & 3 & \\ & 0.0 & 0.0 \\ 50.0 & 0.0\end{array}$




$$
\begin{array}{rrr}
100.000 & -.01000 \\
3 & 3 & \\
& 0.0 & 0.0 \\
50.0 & 0.0 \\
100.0000 & -.010000 \\
4 & 2 & \\
& 0.0 & 0.0 \\
100.0000 & 0.050000 \\
5 & 2 & \\
& 0.0 & 0.0 \\
100.0000 & 0.000000 \\
6 & 2 & \\
& 0.0 & 0.0 \\
100.0000 & 0.000000 \\
7 & 2 & \\
4 & 0.0 & 0.0 \\
100.0000 & 0.000000 \\
8 & 2 & \\
& 0.0 & 0.0 \\
100.0000 & 0.000000 \\
9 & 2 & \\
4 & 0.0 & 0.0 \\
100.0000 & 0.000000
\end{array}
$$




\section{References}

[1] Hallquist, J. O., and Whirley, R. G., DYNASD: Nonlinear Dynamic Analysis of Structures in Three Dimensions, User's Manual, Lawrence Livermore National Laboratory Report UCID-19592, Rev. 5, May, 1989. 


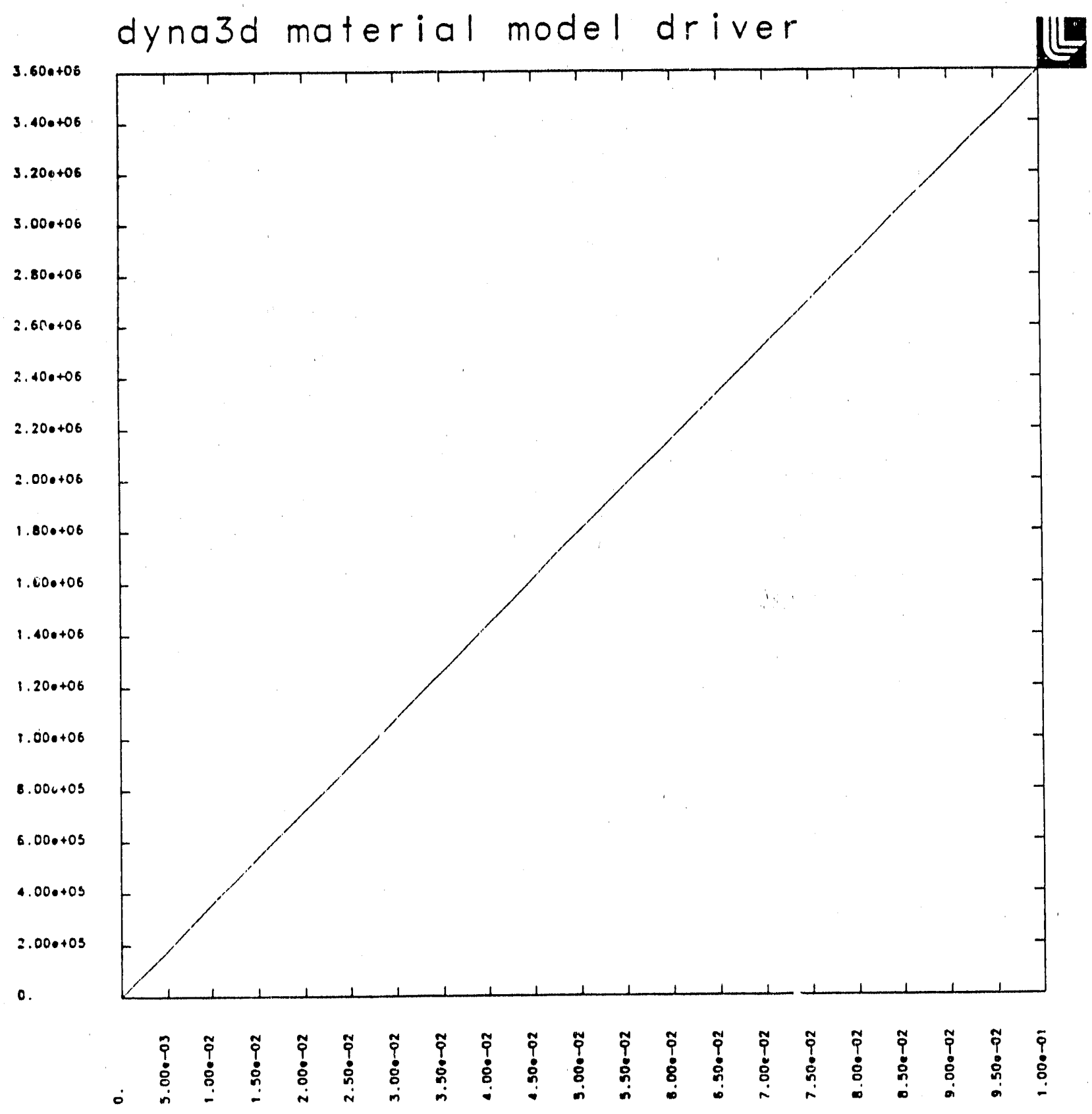

12

Figure 1: Linear Elasticity stress-strain curves in uniaxial strain: $\sigma_{11}$ vs $\varepsilon_{11}$ 


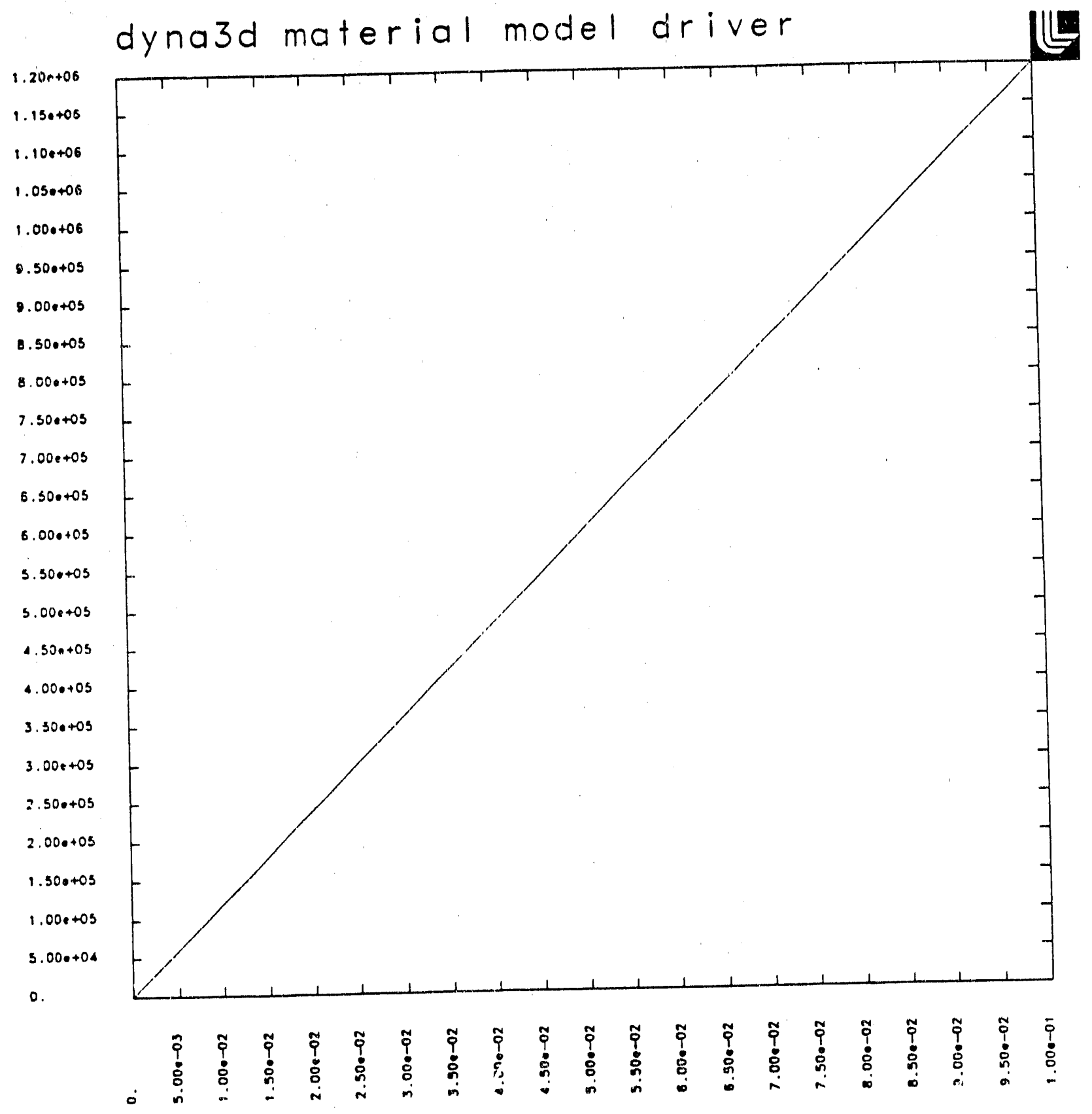

Figure 2: Linear Elasticity stress-strain curves in uniaxial strain: $\sigma_{22}$ vs $\varepsilon_{11}$ 


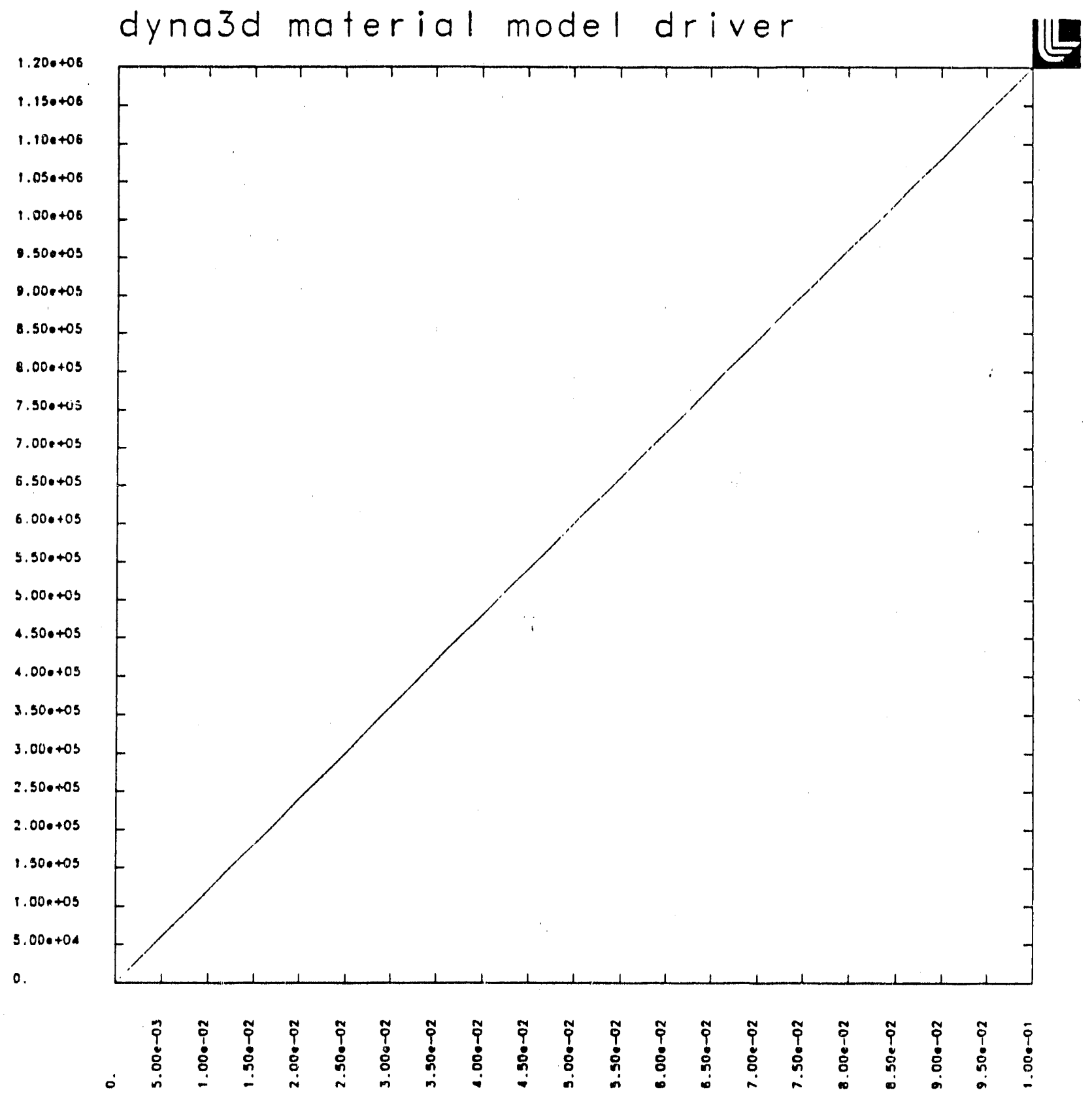

Figure 3: Linear Elasticity stress-strain curves in uniaxial strain: $\sigma_{33}$ vs $\varepsilon_{11}$ 


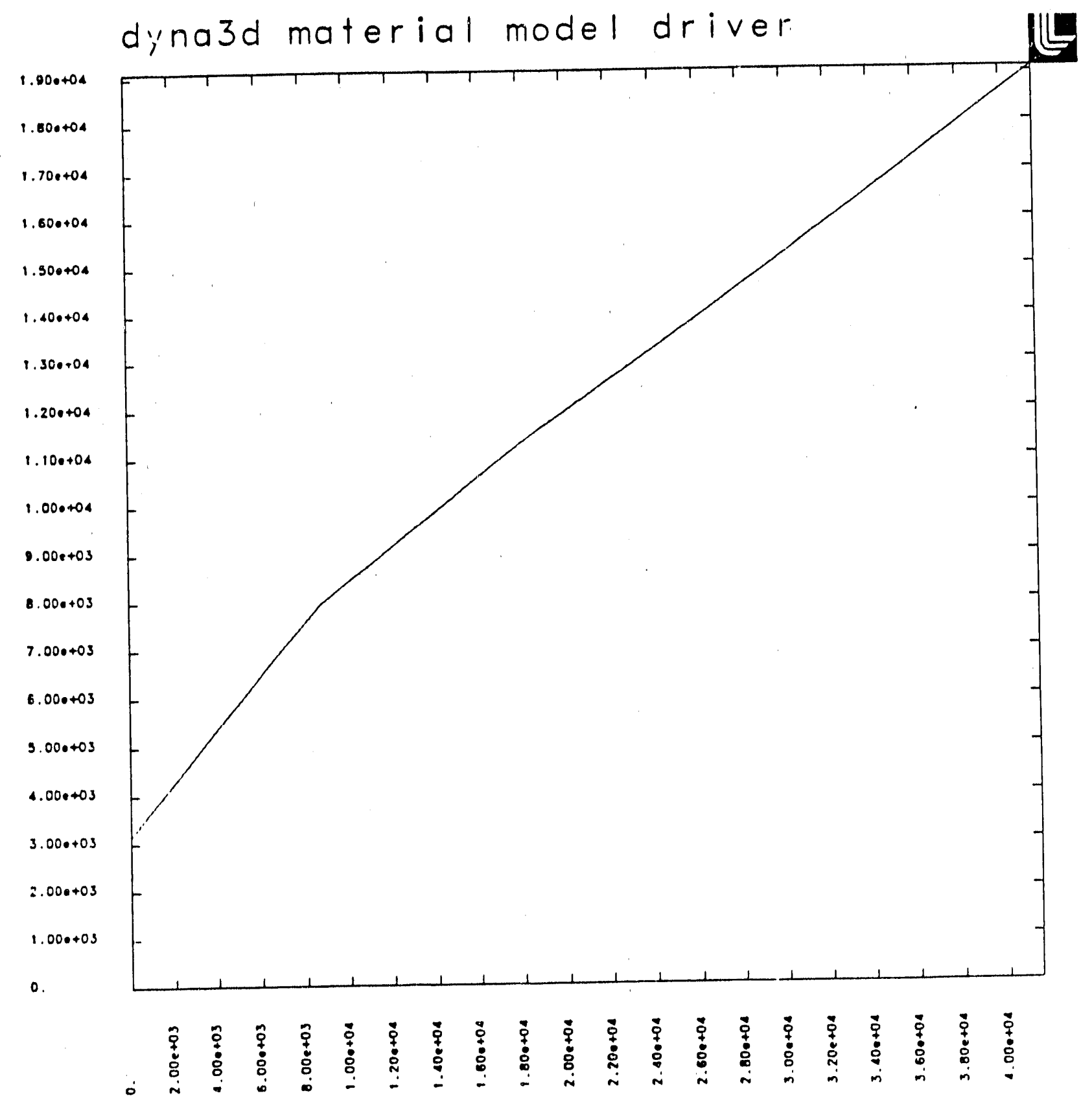

15

Figure 4: Soil and Crushable Foam model: $\sigma_{x y}$ vs $p$ 


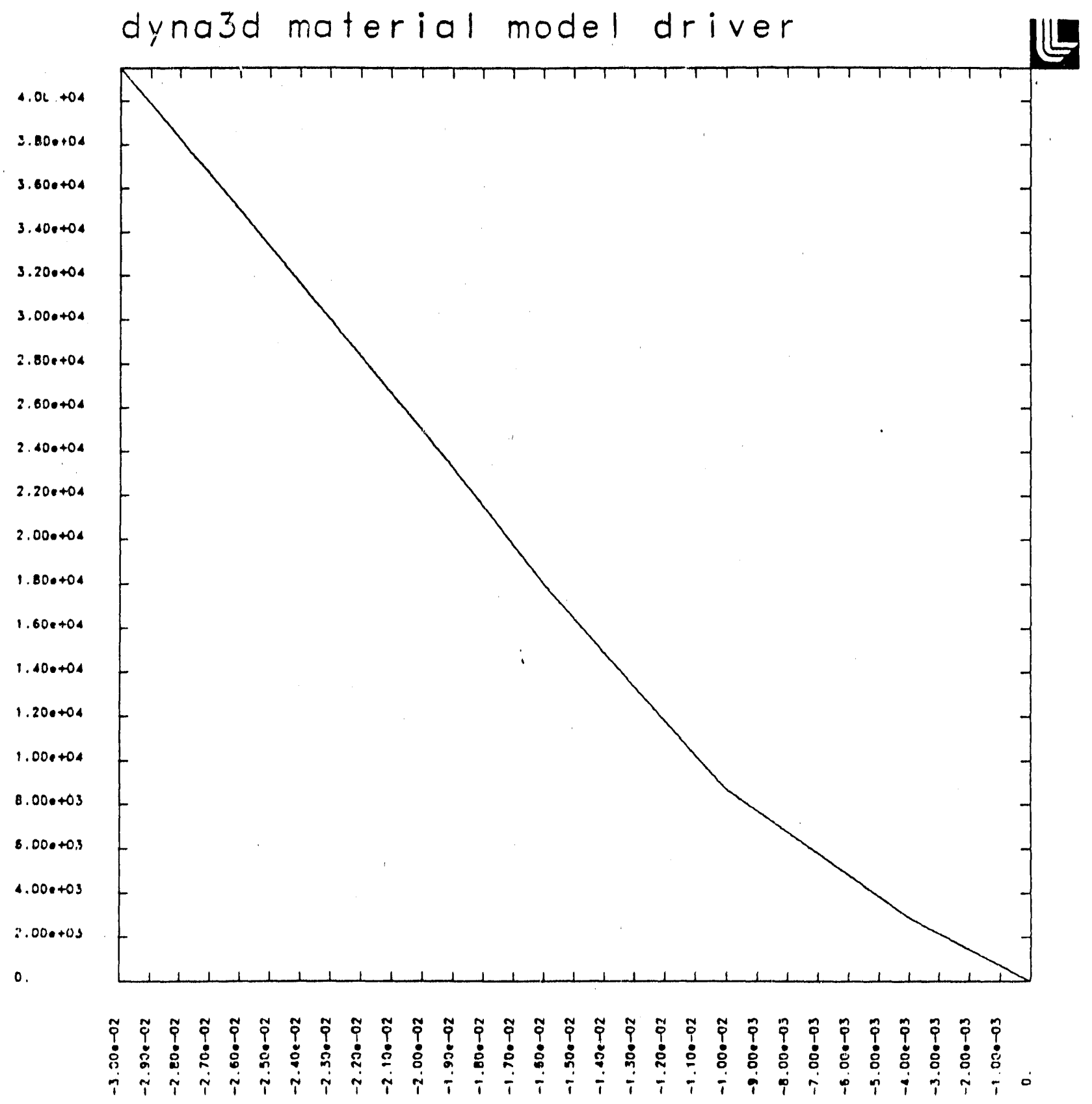

Figure 5: Soil and Crushable Foam model: $p$ vs $\ln \left(\frac{\nu}{v_{0}}\right)$ 


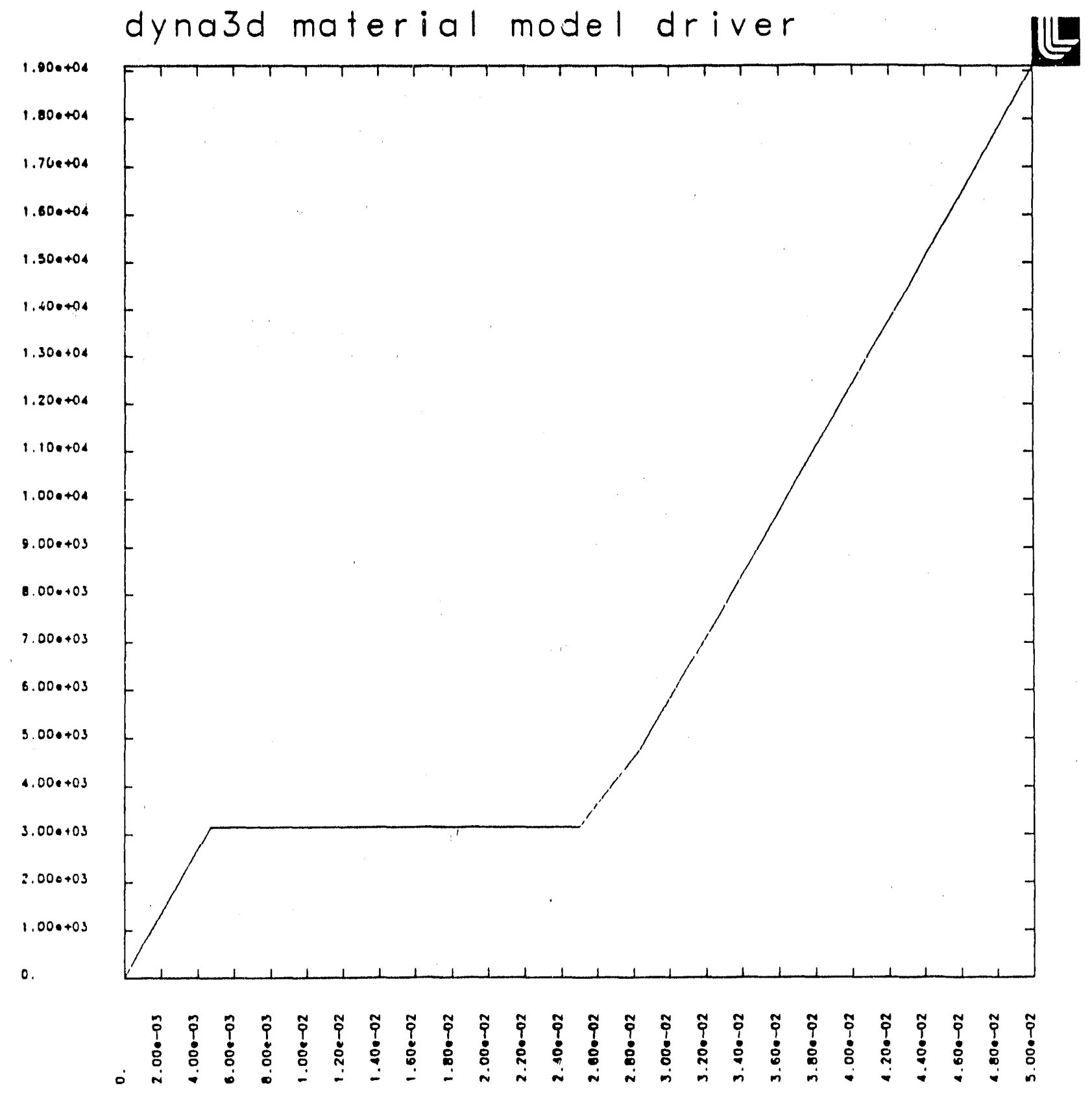

Figure 6: Soil and Crushable Foam model: $\sigma_{x y}$ vs $\gamma_{x y}$ 

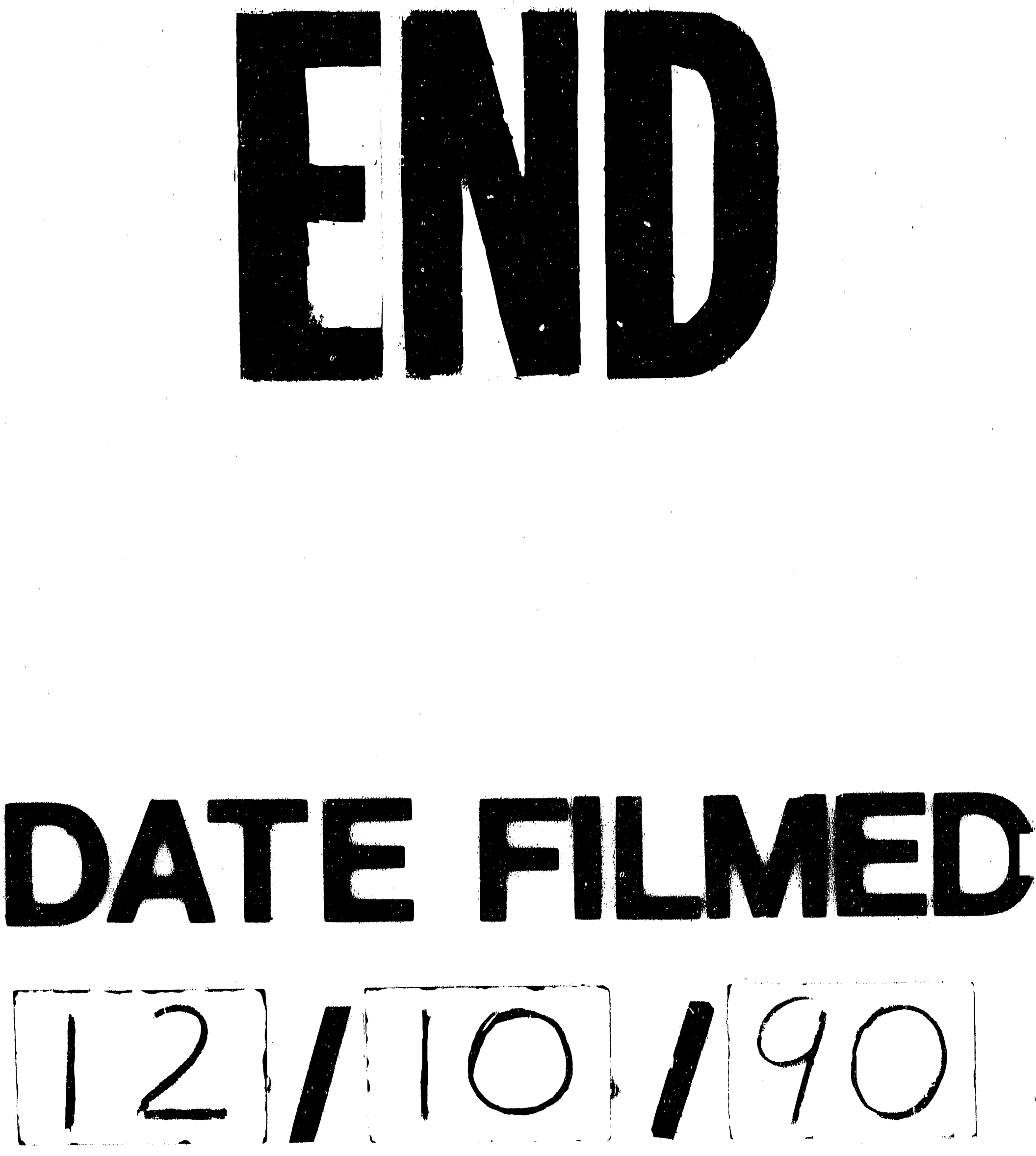


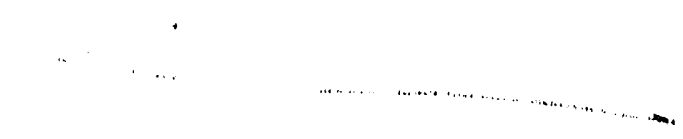

